\title{
The MASIV Legacy: Surveying AGN Intra-day Variability at Radio Wavelengths
}

\author{
J. Y. Koay ${ }^{1,2 *}$, J.-P. Macquart ${ }^{2}$, B. J. Rickett ${ }^{3}$, H. E. Bignall ${ }^{2}$, \\ D. L. Jauncey ${ }^{4}$, T. Pursimo ${ }^{5}$ C. Reynolds ${ }^{2}$, J. E. J. Lovell ${ }^{6}$, \\ L. Kedziora-Chudczer ${ }^{7}$ and R. Ojha ${ }^{8}$ \\ ${ }^{1}$ Dark Cosmology Centre, Niels Bohr Institute, University of Copenhagen, Denmark \\ *email: koayjy@dark-cosmology.dk \\ ${ }^{2}$ International Centre for Radio Astronomy Research, Curtin University, Australia \\ ${ }^{3}$ Department of Electrical \& Computer Engineering, University of California, San Diego, USA \\ ${ }^{4}$ CSIRO Astronomy \& Space Science, \& Australian National University, Australia \\ ${ }^{5}$ Nordic Optical Telescope, Spain \\ ${ }^{6}$ School of Mathematics \& Physics, University of Tasmania, Australia \\ ${ }^{7}$ School of Physics \& Astrophysics, University of New South Wales, Australia \\ ${ }^{8}$ Institute for Astrophysics \& Computational Sciences, Catholic University of America, USA
}

\begin{abstract}
The Micro-arcsecond Scintillation-Induced Variability (MASIV) Survey and its followup observations have provided large datasets of AGN intra-day variability (IDV) at radio wavelengths. These data have shown that IDV arises mainly from scintillation caused by scattering in the ionized interstellar medium (ISM) of our Galaxy, based on correlation with Galactic latitudes and line-of-sight Galactic electron column densities. The sensitivity of interstellar scintillation (ISS) towards source angular sizes has provided a new tool for studying the most compact components of radio-loud AGNs at microarcsecond ( $\mu$ as) scale resolution - much higher than any ground-based radio interferometer. We present here key results from the MASIV Survey and its follow-up observations, and point to relevant papers where these results have been published.
\end{abstract}

Keywords. galaxies: active, radio continuum: ISM, scattering

\section{Background: AGN Intra-day Variability at Radio Wavelengths}

In the last few decades, it has been established that the intra-day variability (IDV) of compact Active Galactic Nuclei (AGNs) at cm-wavelengths is predominantly due to interstellar scintillation (ISS). The idea was first proposed because intrinsic variability on the timescales observed implied brightness temperatures well over the $10^{12} \mathrm{~K}$ inverse Compton limit for incoherent synchrotron emission. Substantial observational evidence has accumulated to support this theory (see Section 2.3 in Lazio et al. (2004) and references therein). The Micro-Arcsecond Scintillation-Induced Variability (MASIV) Survey, conducted from 2002 to 2003, for the first time allowed the phenomenon to be studied for a large sample of $\sim 500$ compact AGNs.

\section{The MASIV Survey and Follow-Up Observations}

MASIV 5 GHz VLA Survey: About 500 flat-spectrum sources, selected from the CLASS and JVAS catalogues, were observed with the Very Large Array (VLA) at $5 \mathrm{GHz}$ in four epochs of 72 hours each, at three-month intervals (Lovell et al. 2003). The VLA was divided into 5 sub-arrays observing different sources, with each sub-array observing $\sim 6$ one-minute scans per source per day.

VLBI follow-up: 75 sources from the MASIV survey were observed at $8.4 \mathrm{GHz}$ in January 2003 using the Very Long Baseline Array (VLBA) (Ojha et al. 2004a). Another 
49 MASIV sources (28 scintillating and 21 non-scintillating) were observed in February 2003, also using the VLBA, at 0.33, 0.61, 1.6, 2.3, and 8.4 GHz (Ojha et al. 2006).

Dual-frequency VLA follow-up: 140 sources from the MASIV Survey (70 at $z<2$, 70 at $z>2$ ) were observed using the VLA in 2009 (Koay et al. 2011). The VLA was divided into 2 sub-arrays, one observing at $5 \mathrm{GHz}$, another at $8.4 \mathrm{GHz}$. Each source was observed for one minute at 2 hour intervals, for a total duration of 11 days.

Optical follow-up: See Pursimo et al. (2013) and companion paper by Pursimo et al. in these proceedings.

\section{Key Results}

- AGN IDV has a Galactic dependence (Lovell et al. 2008), confirming that IDV is predominantly caused by interstellar scintillation (ISS). The amplitude of the variability shows strong correlation with Galactic latitudes and line-of-sight $\mathrm{H}$-alpha intensities obtained from the Wisconsin H-Alpha Mapper (WHAM) Survey (Haffner et al. 2003).

- Interstellar scattering and scintillation are prevalent and important effects! More than half $(\sim 58 \%)$ of compact, flat-spectrum radio-loud AGNs scintillate in at least one of the MASIV epochs (Lovell et al. 2008).

- AGN ISS is also dependent on intrinsic source properties. ISS is dependent on source angular sizes; extended sources scintillate less than compact sources. Follow-up VLBI observations show that ISS increases for sources with higher core-dominance and smaller overall angular extent on mas scales (Ojha et al. 2004b). Sources with steeper spectra $\left(\alpha_{4.9}^{8.4}\right.$ where $\left.S \propto \nu^{\alpha}\right)$ scintillate less than sources with flatter or inverted spectra (Koay et al. 2011); emissions from the latter are more strongly dominated by the optically thick, compact core. ISS thus allows AGNs to be probed at micro-arcsecond scales, much higher than any ground based interferometer.

- The fraction of scintillating sources, as well as their scintillation amplitudes, have been found to decrease significantly for sources with redshifts $z \gtrsim 2$ (Lovell et al. 2008). We determined that the redshift dependence of AGN ISS is partially linked to the steepening of source spectral indices $\left(\alpha_{4.9}^{8.4}\right)$ with redshift, caused either by selection biases or AGN evolution, coupled with weaker ISS in the steeper spectrum sources. Selecting only the $-0.4<\alpha_{4.9}^{8.4}<0.4$ sources, we find that the redshift dependence of ISS is still significant, but is not significantly steeper than the expected $(1+z)^{0.5}$ scaling of source angular sizes due to cosmological expansion, for a brightness temperature and flux-limited sample of sources (Koay et al. 2012).

Acknowledgments: The Dark Cosmology Centre is funded by the Danish National Research Foundation. JYK is supported by a research grant (VKR023371) from VILLUM FONDEN.

\section{References}

Haffner, L. M., et al. 2003, ApJS, 149, 405

Koay, J. Y., et al. 2011, AJ, 142, 108

Koay, J. Y., et al. 2012, ApJ, 746, 29

Lazio, T. J. W., et al. 2004, New Astron. Rev., 48, 1439

Lovell, J. E. J., et al. 2003, AJ, 126, 1699

Lovell, J. E. J., et al. 2008, ApJ, 689, 108

Ojha, R., et al. 2004a, AJ, 128, 1570

Ojha, R., et al. 2004b, ApJ, 614, 607

Ojha, R., et al. 2006, ApJS, 166, 37

Pursimo, T., et al. 2013, ApJ, 767, 14 